\title{
Gaia: A Stereoscopic Census of Our Galaxy
}

\section{T. Prusti}

European Space Agency, ESTEC, The Netherlands

\begin{abstract}
Gaia is a space astrometry mission, a broad survey project following the measurement and operational principles of Hipparcos. It will help solving one of the most difficult yet deeply fundamental challenges in modern astronomy: to create an extraordinarily precise threedimensional map of about one billion stars throughout our Galaxy and beyond. In the process, it will map their three-dimensional motions, which encode the origin and subsequent evolution of the Galaxy. Through comprehensive photometric and spectroscopic classification, it will provide the detailed physical properties of each star observed: characterising their luminosity, temperature, gravity, and elemental composition. This massive stellar census will provide the basic observational data to tackle an enormous range of important problems related to the origin, structure, and evolutionary history of our Galaxy.
\end{abstract}

Within the Galactic plane the specific issue for Gaia is not surprisingly the high stellar density. While the astrometric instrument is expected to detect individually sources separated by no more than 0.1 arcsec, the photometric and spectroscopic instruments will suffer from crowding in the densest parts of the sky. This is due to the fact that the light is dispersed on the CCD. The maximum star densities are 1,000,000, 750,000, and 36,000 stars per square degree for astrometric, photometric and spectroscopic instruments respectively. In principle it is possible to operate Gaia in so called high density mode which will enable better completeness level even in regions with densities above a million stars per square degree. However, to achieve this a modification of the scanning law is required with a negative impact on overall sky homogeneity coverage. The precise use of the high density mode will be decided during the operational phase in order to balance the scientific benefits of an unbiased survey with the nominal scanning law and a better completeness in high density regions. As far as the large data quantities associated with the Galactic Plane are concerned, the planning of ground station time has been tuned to take this into account. When an operational day will contain a large fraction of Galactic Plane, Gaia will be served by two ground stations instead of a single one. This will ensure Gaia gathering a unique data set also for the Galactic Plane.

Currently Gaia is in the development phase with flight hardware being produced and the Data Processing Consortium preparing the software for handling the data. Gaia is scheduled for launch in spring 2012 with intermediate data releases anticipated a few years after the launch and final catalogue 2020. 